\title{
ANÁLISE DOS ÍNDICES DE SAÚDE BUCAL ASSOCIADOS A INDICADORES SOCIAIS E ECONÔMICOS NO BRASIL DE 1986 A 2010
}

\section{ORAL HEALTH INDEX ASSOCIATED WITH SOCIOECONOMIC INDICATORS IN BRAZIL FROM 1986 TO 2010}

Lara Jansiski Motta Programa de Mestrado em Gestão de Sistemas de Saúde da UNINOVE - SP larajmotta@terra.com.br

Adriana Vieira da Silva Pissinato Universidade Nove de Julho - SP adrianapissinato@hotmail.com

Marcelo Mendes Pinto Universidade Nove de Julho - SP mmpdent@hotmail.com

Sonia Francisca Monken Programa de Mestrado em Gestão de Sistemas de Saúde da UNINOVE - SP soniamonken@gmail.com

Submissão: $24 / 09 / 2014$ Aprovação: 25/06/2015 


\title{
RESUMO
}

Os indicadores sociais e econômicos influenciam o processo saúde doença, o que ressalta a importância de relacionar as condições socioeconômicas ao gerenciamento dos serviços de saúde e às condições de saúde da população. Diante disso o presente estudo tem como objetivo avaliar a relação entre variáveis socioeconômicas e a condição de saúde bucal da população brasileira, considerando os levantamentos nacionais de saúde bucal realizados em 1986, 1996, 2003 e 2010. Os indicadores de qualidade de vida avaliados foram expectativa de vida; PIB (Produto Interno Bruto) per capita e escolaridade. Análises como esta se tornam importantes, para que seja interpretado de maneira macro, a situação do país para entendermos o papel das políticas públicas na saúde, além de refletir na importância da informação e acesso a serviços para controle de doenças que podem ser prevenidas, como é o caso da cárie.

Palavras-chave: Expectativa de vida. Índices de saúde bucal. Indicadores socioeconômicos.

\begin{abstract}
Social and economics indicators influence health disease process, this situation warns the importance of the relationship between socioeconomic management of health services and the health conditions. This study aims to evaluate the relationship between socioeconomics variables and the oral health status of the brazilian population, considering the national oral health surveys conducted in 1986, 1996, 2003 and 2010; life expectance; Gross Domestic Product and education. This analysis is important, to understand the situation of the country and the public policies in health, in addition to consider the importance of access to information and services to control the diseases that can be prevented, as in the case of caries.
\end{abstract}

Keywords: Life Expectance. Oral Health Index. Socioeconomics Indicators. 


\section{INTRODUÇÃO}

A saúde e o desenvolvimento socioeconômico estão intensamente relacionados, considerando que o desenvolvimento se dá pela consolidação do direito à saúde. Nessa visão, parte-se da premissa de que o desenvolvimento é multidimensional e que não deve-se considerar apenas a dimensão biológica, apenas a dimensão social ou a econômica, mas o conjunto de indicadores situacionais. Os indicadores influenciam e sofrem influência das características sociais, culturais e políticas que compõem qualidade de vida humana. Diante disso, uma das condições para o desenvolvimento com equidade é a saúde, sendo considerada como fator decisivo para o bem-estar do cidadão, das famílias e da comunidade (CARRARA; VENTURA, 2012).

A saúde de uma comunidade depende da qualidade e do acesso ao consumo de bens e de serviços como habitação, alimentação, educação, trabalho e serviços de saúde (JUNQUEIRA et al., 2006).

Os levantamentos epidemiológicos contribuem na avaliação de riscos e ocorrência de doenças em determinada população, possibilitando a compreensão de fatores da biologia humana, relacionados a estilo de vida e serviços de saúde. Os agravos bucais e suas sequelas são de grande prevalência no Brasil, constituindo-se em problemas de saúde pública, com graves consequências sociais e econômicas. As pesquisas que investigam indicadores de saúde bucal e condições sociais e econômicas são particularmente importantes para a formulação de políticas de saúde direcionadas a reduzir desigualdades em saúde, sobretudo no acesso e utilização dos serviços (FERNANDES; PERES, 2005).

A literatura demonstra a influência dos fatores socioeconômicos e comportamentais no desenvolvimento das doenças bucais, tanto no que diz respeito a prevalência quanto à incidência da doença. Assim sendo, a avaliação de indicadores socioeconômicos é de grande relevância nos estudos que envolvem a saúde da sociedade. Esta análise permite descrever a situação da população através do conhecimento de suas condições de vida.

Até recentemente, poucos estudos investigaram a relação entre as condições bucais e seu impacto na vida das pessoas e nas condições sociais, mas na última década, houve aumento do interesse em quantificar as consequências das doenças (MINAYO; HARTZ; BUSS, 2000).

Frente às representações sociais e epidemiológicos brasileiras, considerando-se que a qualidade de vida possa ser avaliada mediante indicadores ligados à instrução, renda e desigualdade em sua distribuição, entre outros, as políticas públicas precisam ser direcionadas 
para a promoção da saúde. Suas propostas devem ressaltar a criação de condições adequadas ao desenvolvimento da saúde e competência dos indivíduos, o que necessita de uma abordagem intersetorial, em conjunto com ações e serviços na área da Saúde. (BUSS, 2000).

Desigualdade social em saúde refere-se às diferenças produzidas socialmente na qualidade de vida e na capacidade de ser e agir dos grupos sociais e indivíduos. A saúde de uma população, depende da qualidade e do acesso ao consumo de bens e de serviços de subsistência, como moradia, alimentação, educação, trabalho e assistência à saúde; portanto, diferenças no acesso a esses bens e serviços resultam na desigualdade social em saúde (DRACHLER et al., 2003).

A evolução favorável de uma série de indicadores sociais no Brasil dos anos 80 esteve relacionada à ampliação dos dispêndios e à transformação nas formas de implementação das políticas públicas de saúde e nutrição, o que coincide com a criação do Sistema Único de Saúde (SUS) no País, em 1988.

$\mathrm{O}$ acesso a bens e serviços essenciais à sobrevivência ainda depende da possibilidade de pagar por eles. Embora a noção de bem-estar não se restrinja ao fator renda, é inegável sua importância para uma sociedade como esta. A renda média da população do Estado mais rico do País foi de 2,2 salários mínimos, em que $8,0 \%$ da população vivia com renda insuficiente (até meio salário mínimo) e quase metade das pessoas dispunham de até três salários mínimos.

Os estudos epidemiológicos contribuem para a avaliação da ocorrência de doenças em determinadas comunidades, ampliando a compreensão de fatores como biologia humana, ambiente, estilo de vida e serviços de saúde, que relacionam-se ao processo saúde-doença. A saúde descreve os efeitos adversos para a qualidade de vida, analisa a relação entre exposição a fatores sociais com índices socioeconômicos e contribui para a prevenção de efeitos antagônicos. A Epidemiologia analisa situações de riscos como saneamento, água para consumo humano, pobreza, equidade, poluição química, condições psicossociais, assim como a necessidade de desenvolvimento da população (SILVA et al., 2006).

A cárie dentária é uma doença crônica que acomete a estrutura dental. Mesmo com o declínio dos seus índices na população infantil, esta continua sendo o principal problema de saúde bucal na maioria dos países industrializados e importante causa de dor e perda dentária (SHEIHAM, 2005). Esta situação pode levar ao absenteísmo no trabalho e na escola e comprometer o desempenho das atividades diárias e a estabilidade emocional dos indivíduos. A análise do retrato de que a melhoria na saúde bucal entre crianças tem se desdobrado para a população adulta é relevante (SKUDUTYTE-RYSSTAD; ERIKSEN, 2007). O estado de 
saúde bucal entre 35 e 44 anos de idade reflete o histórico das políticas de prevenção e tratamento. Apesar do declínio dos índices de cárie na população infantil no Brasil, esta situação não é observada nos adultos. Três levantamentos epidemiológicos nacionais colheram dados da população com idade de 35 a 44 anos, mas os resultados produzidos ainda não foram comparados. Essa análise pode sugerir tendências que permitem explorar conexões entre as taxas de ocorrência dessa doença ao longo do tempo e aspectos mais gerais, ligados à industrialização, ao desenvolvimento humano e às políticas nacionais de saúde bucal (SKUDUTYTE-RYSSTAD; ERIKSEN, 2007).

Estudos transversais de abrangência nacional, planejados e financiados pelo Ministério da Saúde, foram realizados, e nestes levantamentos foram analisados os índices de cárie adultos de 35 a 44 anos de idade e crianças de 12 anos, em 1986, 2003 e 2010, e em 1996 o estudo avaliou apenas as crianças de 12 anos, em residentes das cinco regiões brasileiras.

Buchwald et al,. (2013) em estudo na Alemanha observaram que o nível de escolaridade estava relacionado com índice de perda dental, demonstrando que a baixa renda e baixa escolaridade aumentavam em duas vezes o risco de perda dental. Outro estudo europeu, realizado em 2009 e publicado em 2013 analisou as diferenças socioeconômicas regionais e concluiu que em locais em que as desigualdades educacionais e sociais eram maiores, o índice de edentulismo também era maior (GUARNIZO-HERREÑO et al., 2013).

No Brasil, um estudo realizado em Minas Gerais, analisou a associação entre proporção de exodontias, indicadores socioeconômicos e oferta de serviços odontológicos. Os fatores socioeconômicos, como distribuição de renda, e de organização dos serviços de saúde bucal explicam grande parte da mutilação dentária nessa região (PALMIER et al., 2012).

A economia brasileira tem crescido vigorosamente nos últimos anos e os indicadores sociais brasileiros também melhoraram; no entanto, a desigualdade regional ainda é importante. Os contrastes de desenvolvimento humano no Brasil ficam evidentes quando se observam as mudanças no IDH em cada região (BACEN, 2013).

A realização de estudos que avaliem a associação entre indicadores socioeconômicos e indicadores de atenção primária à saúde bucal é importante para subsidiar a formulação de políticas de saúde que tenham como objetivo reduzir desigualdades em saúde, sobretudo no que diz respeito ao acesso e à utilização de serviços.

Diante disso, o presente trabalho tem como objetivo realizar uma análise das condições de saúde bucal e as relações com indicadores socioeconômicos, comparando os dados dos levantamentos epidemiológicos nacionais de saúde bucal de 1986, 1996, 2003 e 2010 e a expectativa de vida, PIB per capita e escolaridade no Brasil nos mesmos anos 
índices. Para tanto, realizou-se um estudo empírico analítico de análise documental, com abordagem quantitativa dos dados.

\section{PROCEDIMENTOS METODOLÓGICOS}

Este estudo é de natureza documental, descritiva e analítica. O trabalho analisou informações de levantamentos epidemiológicos nacionais do Ministério da Saúde (Brasil) e dados sociais e econômicos do banco de dados do IPEADATA. Essas características oferecem uma análise de dados documentais para que se descreva a condição de saúde bucal no Brasil e a situação social e econômica em períodos específicos entre 1986 e 2010.

A pesquisa é classificada como documental com utilização de fontes primárias. Documentos de fontes primárias são aqueles de primeira mão, provenientes dos próprios órgãos que realizaram as observações. Englobam todos os materiais, ainda não elaborados, escritos ou não, que podem servir como fonte de informação para a pesquisa científica e ser encontrados em arquivos públicos ou particulares, ou em fontes estatísticas compiladas por órgãos oficiais e particulares (THEÓPHILO; MARTINS, 2009).

Os dados coletados foram referentes ao índice de cárie, medido pelo CPOD (número de dentes cariados, perdidos e restaurados) e pelos indicadores expectativa de vida; PIB per capita (PPC) e escolaridade em anos de estudo. Foi considerada como variável dependente o índice de saúde bucal e como variáveis independentes os indicadores socioeconômicos. Apenas para a análise da relação entre o índice de cárie e a expectativa de vida realizou-se uma análise em particular, em que o desfecho final é a variável expectativa de vida.

A análise seguiu dois procedimentos, sendo um relacionado à avaliação do índice de cárie ao 12 anos nos anos de 1986, 1996, 2003 e 2010 e a relação com os indicadores socioeconômicos e outro contemplando a avaliação do índice CPOD entre 35 e 44 anos de idade em 1986, 2003 e 2010. Os índices foram analisados e representados graficamente em tabelas descritivas. Para análise da correlação entre as variáveis estudadas utilizou-se o teste de correlação de Pearson. Os dados foram tabulados e analisados no programa estatístico IBM SPSS for win 21.

\section{ANÁLISE E DISCUSSÃO DOS RESULTADOS}

A tabela 1 descreve a distribuição dos índices de cárie e os índices socioeconômicos nas datas em que foram realizados os levantamentos nacionais de saúde bucal. Os índices 
apresentados aos 12 e dos 35 a 44 anos, estão relacionados à doença cárie. Observa-se que nas duas faixas etárias houve uma redução no índice de CPOD, que representa a média populacional do número de dentes comprometidos pela cárie, estando cariados, perdidos ou obturados. No ano de 1996 não foram incluídos na avaliação os adultos com idade entre 35 e 44 anos, neste caso os levantamentos nacionais permitem a comparação dos dados deste ano apenas na faixa dos 12 anos.

\section{Tabela 1}

Descrição da condição de saúde bucal e indicadores socioeconômicos brasileiros entre 1986 e 2010.

\begin{tabular}{ccccc}
\hline Ano & $\begin{array}{l}\text { Índice saúde bucal } \\
\text { (CPOD) aos 12 anos }\end{array}$ & $\begin{array}{l}\text { Índice saúde bucal } \\
\text { (CPOD) entre 35 e } \\
\text { 44anos }\end{array}$ & $\begin{array}{c}\text { PIB per } \\
\text { capita PPC } \\
\text { (U\$) }\end{array}$ & $\begin{array}{c}\text { Escolaridade em } \\
\text { anos de estudo }\end{array}$ \\
\hline $\mathbf{1 9 8 6}$ & 6,65 & 22,5 & 4752,65 & 4,4 \\
\hline $\mathbf{1 9 9 6}$ & 3,06 & - & 6427,34 & 5,4 \\
\hline $\mathbf{2 0 0 3}$ & 2,8 & 20,13 & 7522,06 & 6,3 \\
\hline $\mathbf{2 0 1 0}$ & 2,1 & 16,3 & 11180,29 & 7,2 \\
\hline
\end{tabular}

Fonte: Ministério da Saúde - Disponível em:http://dab.saude.gov.br/CNSB/sbbrasil/ IPEADATA - Disponível em < http://www.ipeadata.gov.br/>.

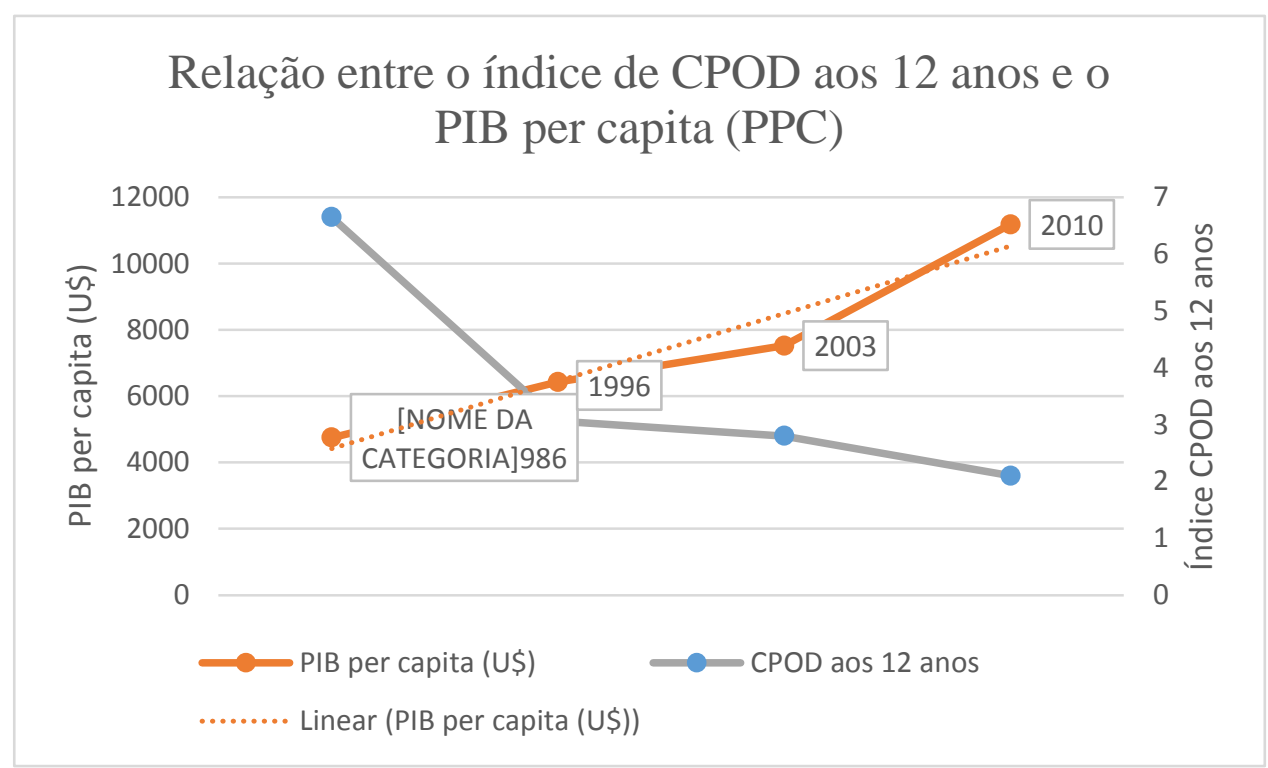

Figura 1. Representação gráfica da relação entre o índice CPOD aos 12 anos e o PIB per capita nas datas índices dos levantamentos nacionais. 
Relação entre o índice de CPOD aos 12 anos e

escolaridade em anos de estudo

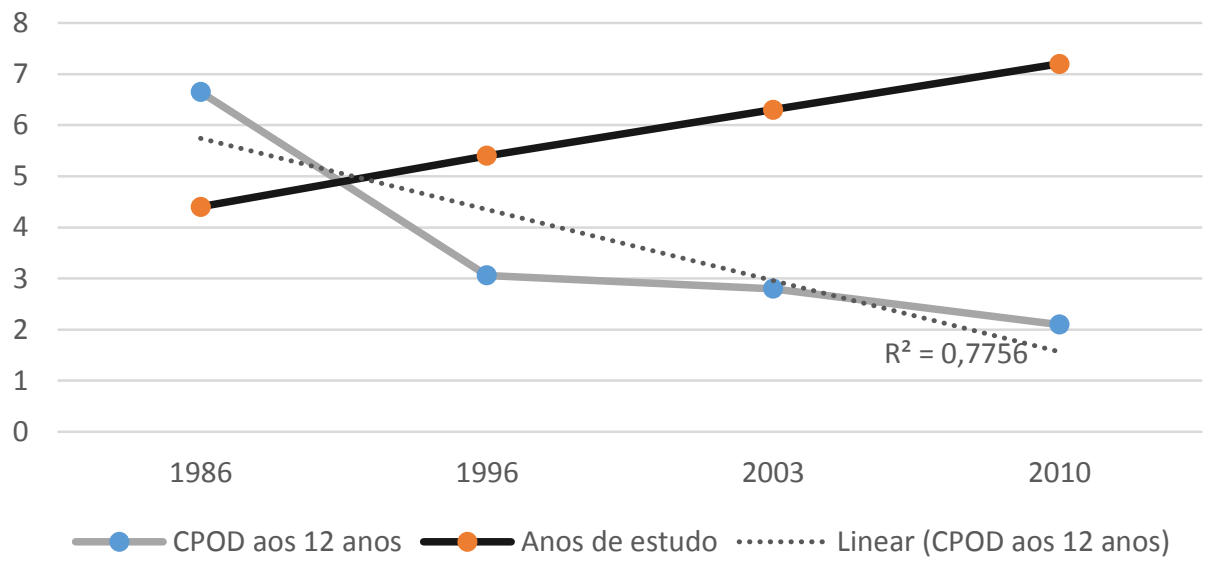

Figura 2. Representação gráfica da relação entre o índice CPOD aos 12 anos e escolaridade nas datas índices dos levantamentos nacionais.

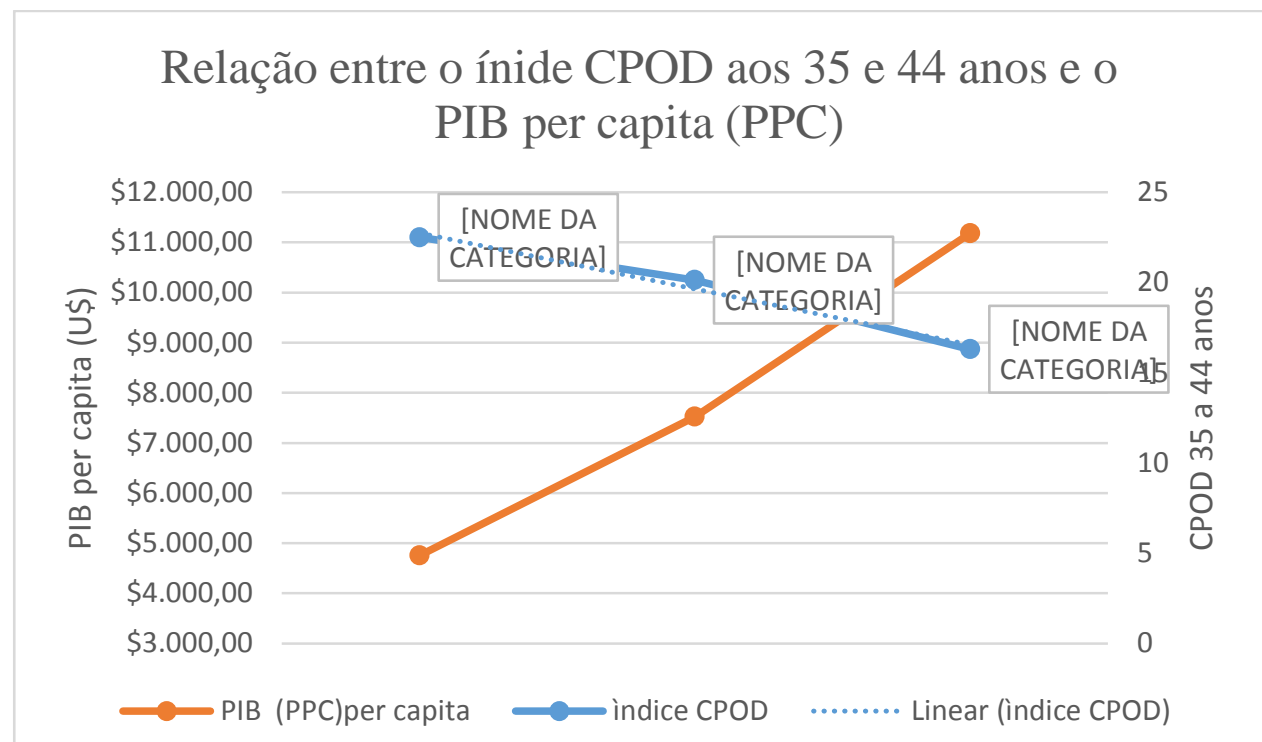

Figura 3. Representação gráfica da relação entre o índice CPOD aos 35 e 44 anos e PIB per capita nas datas índices dos levantamentos nacionais. 


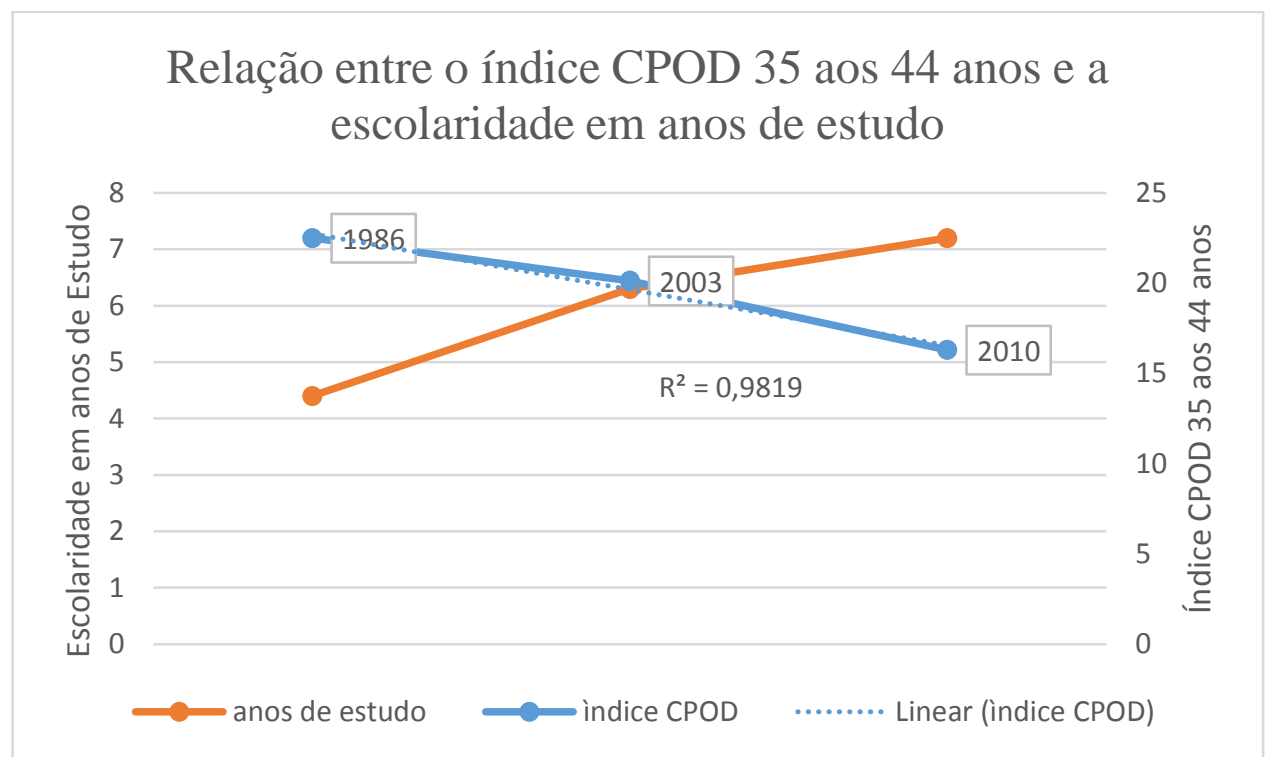

Figura 4. Representação gráfica da relação entre o índice CPOD aos 35 e 44 anos e escolaridade nas datas índices dos levantamentos nacionais.

As figuras 1 e 2 representam a relação entre os índices de cárie CPOD aos 12 anos ao longo do período estudado e as variáveis PIB per capita (PPC) e escolaridade em anos de estudo. Segundo Almeida et al. (2007) observa-se um avanço na oferta dos serviços de saúde desde as décadas de 40 e 50, notando-se uma tendência de melhora até os dias de hoje nas condições de saúde dos brasileiros. A cárie dentária também acompanha esta tendência no que diz respeito à diminuição do seus índices, principalmente na população infantil. O declínio do índice, apesar de observado, não é considerado baixo para a faixa etária de 35 a 44 anos, como é possível observar nas figuras 3 e 4 . No entanto os índices apresentados neste artigo representam o retrato nacional e o Brasil é marcado por desigualdades socioeconômicas, o que determina extensas consequências para a saúde, inclusive para o acesso dos serviços odontológicos. Apesar de apresentar um declínio no índice de cárie no Brasil de 1986 a 2010, este índice não é distribuído de maneira homogênica pelo país, assim como a distribuição de renda, acesso aos serviços e a escolaridade também se apresentam de maneira pouco igualitária no território nacional. Ao analisar os índices como um dado nacional, sem considerar as desigualdades, observa-se que o aumento do PIB per capita entre 1986 e 2010, apresentou forte correlação com a diminuição do índice de CPOD, tanto aos 12 anos quanto na fase adulta (de 35 a 44 anos), sendo considerada esta correlação estatisticamente significante na fase adulta, conforme expresso na tabela 2. A representação da maior aquisição de serviços pelo PIB per capita ao longo dos anos, nos faz refletir também na maior possibilidade de acesso da população brasileira aos serviços odontológicos.

Tabela 2. 
Correlação entre os indicadores estudados no período de 1986 a 2010.

\begin{tabular}{clcc}
\hline & \multicolumn{2}{c}{ PIB per capita (PPC) } & Escolaridade \\
\hline $\begin{array}{c}\text { Índice CPOD } \\
\mathbf{1 2} \text { anos }\end{array}$ & $\begin{array}{l}\text { Correlação de } \\
\text { Pearson }\end{array}$ & $-0,800$ & $-0,893$ \\
\hline \multicolumn{1}{c}{ Sig. (2-taled) } & 0,200 & 0,107 \\
\hline \multicolumn{1}{l}{$\begin{array}{c}\text { Índice CPOD } \\
\text { 35 a 44 anos }\end{array}$} & $\begin{array}{c}\text { Correlação de } \\
\text { Pearson }\end{array}$ & $-0,998$ & Escolaridade \\
\hline \multicolumn{2}{c}{ Sig. (2-taled) } & $0,035^{*}$ & $-0,943$ \\
\hline
\end{tabular}

* A correlação é significativa no nível 0,05

Os indicadores de escolaridade, não revelam somente taxa de analfabetismo, são indicadores educacionais. A educação abre espaço para a oportunidade de interação e de acesso à informação. Considerando que a cárie dentária é uma doença que apresenta a etiologia conhecida e seus fatores causadores podem ser controlados, as ações preventivas tornam-se fundamentais para o seu controle. Observou-se neste estudo forte correlação entre escolaridade e a presença da cárie, sendo inversamente proporcional, ou seja, quanto maior a média de anos de estudo da população, menos o índice CPOD. O controle dos fatores etiológicos depende da ação individual, mas para isso o indivíduo precisa receber as orientações corretas em situações que permitam uma integração educacional mais ampla. Estes resultados corroboram com estudos (BALDANI et al., 2002; GONÇALVES et al., 2002) que demonstram forte relação entre os níveis de prevalência de cárie, a baixa renda e a escolaridade.

A saúde bucal afetada, implica em diversos distúrbios para o indíduo, incluindo a fala, o convívio social, comprometendo diretamente a qualidade de vida, além de danos físicos devido à traumas na mastigação, dores recorrentes e a alteração no padrão alimentar que pode acarretar em agravos da saúde geral como doenças crônicas, cardiovasculares, má nutrição e obesidade (FIGUEIREDO et al., 2012). Diante disso, observa-se que a expectativa de vida apresenta uma relação estreita com as condições de saúde. Na tabela 3 é possível observar os dados relacionados à expectativa de vida dos brasileiros em anos no período analisado neste trabalho. Conforme apresentado nas figuras 5 e 6, nota-se que existe uma relação entre o índice de cárie e a expectativa de vida, ou seja, quanto menor o índice da doença maior a expectativa de vida dos brasileiros. Ao analisar a correlação entre estes dados, observou-se forte correlação tanto aos 12 anos $(r=0,983)$ quanto na vida adulta $(r=0,916)$, sendo considerada, neste estudo, estatisticamente significante a correlçao entre o índice de cárie aos 12 anos e o indicador de expectativa de vida. 
Estes resultados levam a refletir que a qualidade de vida das pessoas é prejudicada pelas condições de saúde bucal, não como um único fator, mas por fazer parte da saúde geral, mas também por acarretar agravos de ordem social.

Tabela 3

Descrição da condição de saúde bucal e expectativa de vida dos brasileiros entre 1986 e 2010.

\begin{tabular}{cccc}
\hline Ano & $\begin{array}{c}\text { Índice saúde bucal } \\
\text { (CPOD) aos 12 anos }\end{array}$ & $\begin{array}{c}\text { Índice saúde bucal } \\
\text { (CPOD) entre 35 e } \\
\text { 44anos }\end{array}$ & $\begin{array}{c}\text { Expectativa } \\
\text { de vida (anos) }\end{array}$ \\
\hline $\mathbf{1 9 8 6}$ & 6,65 & 22,5 & 64,82 \\
\hline $\mathbf{1 9 9 6}$ & 3,06 & - & 68,73 \\
\hline $\mathbf{2 0 0 3}$ & 2,8 & 20,13 & 71 \\
\hline $\mathbf{2 0 1 0}$ & 2,1 & 16,3 & 73,1 \\
\hline
\end{tabular}

Fonte: Ministério da Saúde. Disponível em: http://dab.saude.gov.br/CNSB/sbbrasil/; IPEADATA. Disponível em: < http://www.ipeadata.gov.br/>.

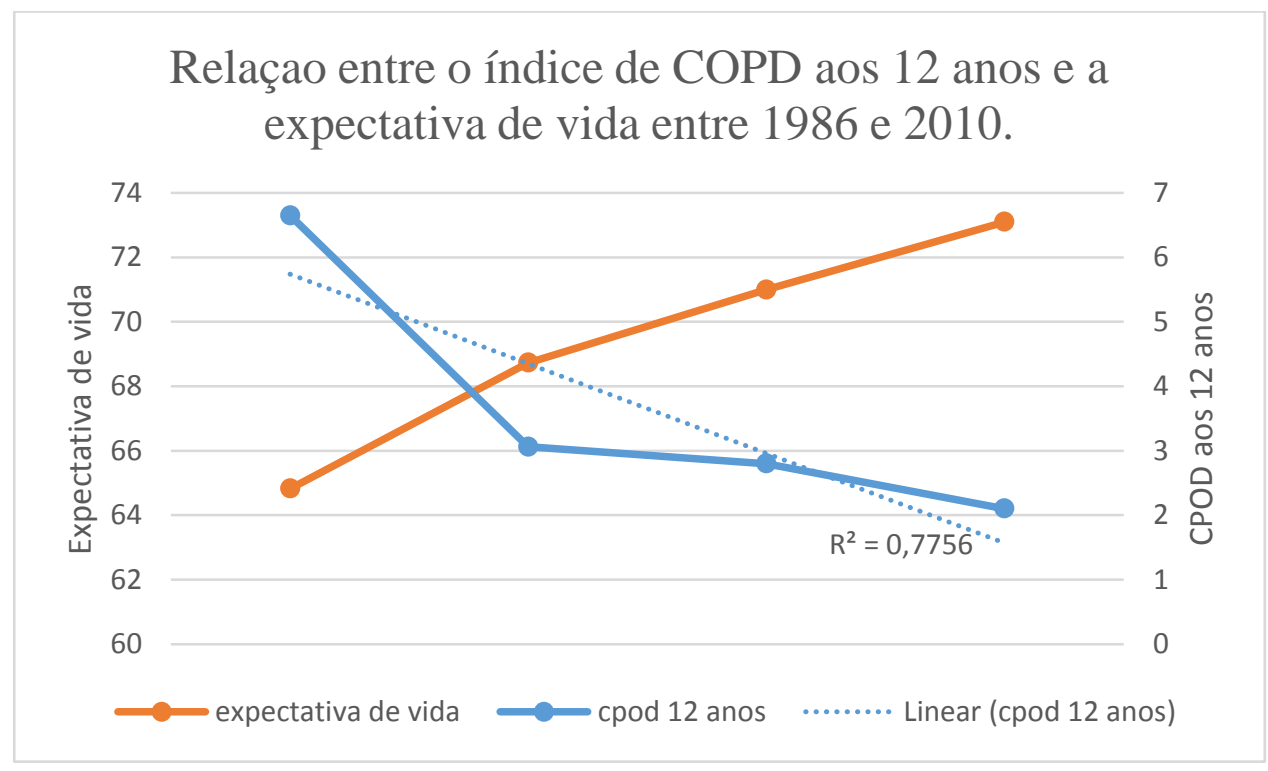

Figura 5. Representação gráfica da relação entre o índice CPOD aos 12 anos e expectativa de vida dos brasileiros. 


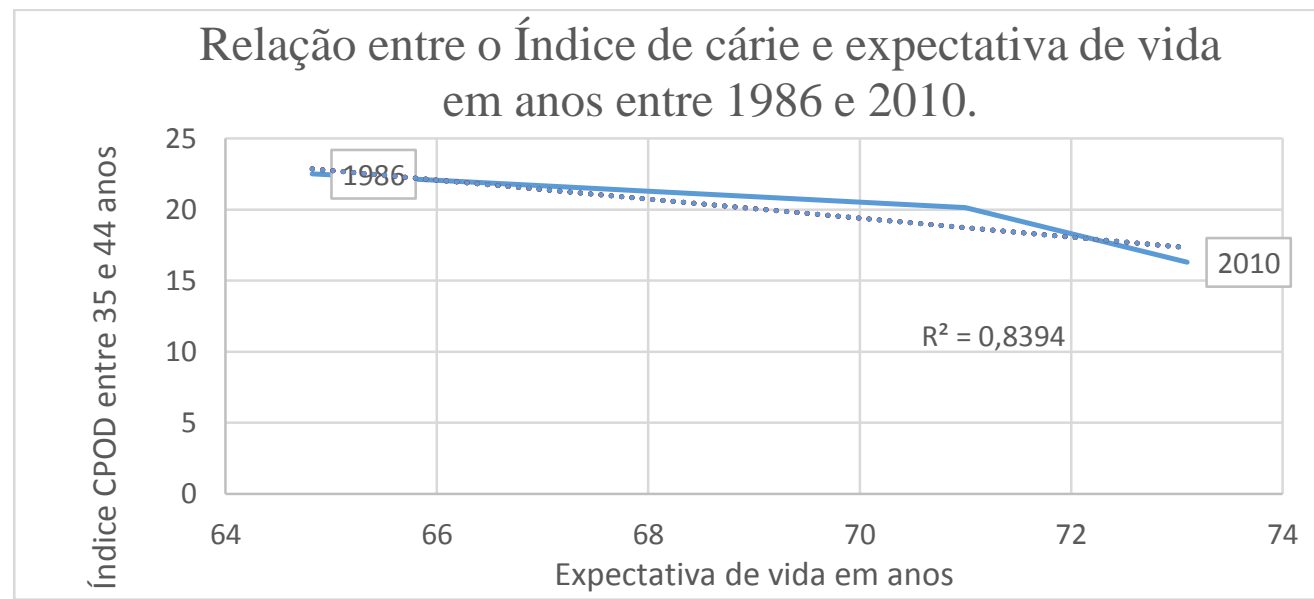

Figura 6. Representação gráfica da relação entre o índice CPOD aos 35 e 44 anos e expectativa de vida dos brasileiros.

\section{Tabela 4}

Correlação entre o índice de saúde bucal e expectativa de vida dos brasileiros entre 1986 e 2010 .

\begin{tabular}{ccc}
\hline & & Expectativa de vida \\
\hline $\begin{array}{c}\text { Índice CPOD } \\
\mathbf{1 2} \text { anos }\end{array}$ & $\begin{array}{c}\text { Correlação de } \\
\text { Pearson }\end{array}$ & $-0,983$ \\
\hline & Sig. $(2$-taled $)$ & $0,047^{*}$ \\
\hline & Expectativa de vida \\
\hline $\begin{array}{c}\text { Índice CPOD } \\
\mathbf{3 5} \text { a 44 anos }\end{array}$ & $\begin{array}{c}\text { Correlação de } \\
\text { Pearson }\end{array}$ \\
\hline & Sig. (2-taled) & $-0,916$ \\
\hline A correlação é significativa no nível 0,05
\end{tabular}

\section{CONSIDERAÇÕES FINAIS}

Este trabalho abordou a análise da relação entre os índices nacionais de saúde bucal no Brasil, representados pelo CPOD e indicadores sociais e econômicos no período de 1986 e 2010. Análises como esta se tornam importantes, para que seja interpretado de maneira macro, a situação do país para entendermos o papel das políticas públicas na saúde, além de refletir na importância da informação e acesso a serviços para controle de doenças que podem ser prevenidas, como é o caso da cárie. Os indicadores estudados são determinados a partir de estruturações econômicas e sociais e podem ser comparados mundialmente. É evidente que a saúde bucal está diretamente relacionada aos fatores sociecoeconomicos da população, assim como ficou claro que a boa condição de saúde bucal influencia na qualidade de vida e longevidade dos indivíduos. Cabe ressaltar que a análise realizada neste artigo, se baseou em dados nacionais que representam a população brasileira de maneira geral e não considerou as 
diferenças sociais e econômicas presentes no Brasil. No entanto, reflexões gerais como esta permitem a conscientização de que é necessário o desenvolvimento de politicas de saúde socialmente orientadas pelas condições da população. Ao buscar direções para a implementação de programas, deve-se analisar os índices da doença, porém, é necessário ir além da perspectiva individual e biológica, é preciso buscar causas mais abrangentes da população geral. Assim, conhecendo o comportamento da interação entre os índices de saúde e os indicadores gerais populacionais, como socioeconômicos e culturais é possível comprrender melhor a realidade e assim poder atingir com maior abrangência a necessidade da população. 


\section{REFERÊNCIAS}

ALMEIDA, Marco Antonio Bettine; GUTIERREZ, Gustavo Luis. Índices de qualidade de vida no Brasil: instrumento para análise crítica dos indicadores e das políticas públicas. Revista Gestão Industrial, v. 3, n. 3, 2007.

BALDANI, Márcia Helena; NARVAI, Paulo Capel; ANTUNES, José Leopoldo Ferreira. Cárie dentária e condições sócio-econômicas no Estado do Paraná. Brasil, 1996, Cad. Saúde Pública, v. 18, n. 3, p. 755-763, 2002.

Brasil. Ministério da Saúde (MS). Projeto SB Brasil2013: condições de saúde bucal da população brasileira. Resultados principais. Brasília: MS;2013. Disponível em: http://dab.saude.gov.br/CNSB/sbbrasil/

Banco Central do Brasil. Banco Central; 2013; 3(1). Disponível em: http://www.bcb.gov.br/?BOLREGIONAL

BUCHWALD, Sabine et al.. Tooth loss and periodontitis by socio-economic status and inflammation in a longitudinal population-based study. Journal of clinical periodontology, v. 40, n. 3, p. 203-211, 2013.

BUSS, Paulo Marchiori. Promoção da saúde e qualidade de vida. Ciência \& saúde coletiva, v. 5, n. 1, p. 163-177, 2000.

CARRARA, Bruna Sordi; VENTURA, Carla Aparecida Arena. A saúde e o desenvolvimento humano. Saúde \& Transformação Social/Health \& Social Change, v. 3, n. 4, p. 89-96, 2012.

DRACHLER, Maria de Lourdes et al. Proposta de metodologia para selecionar indicadores de desigualdade em saúde visando definir prioridades de políticas públicas no Brasil. Ciência $\&$ Saúde Coletiva, v. 8, n. 2, p. 461-470, 2003.

FERNANDES, Liliane Simara; PERES, Marco Aurélio. Associação entre atenção básica em saúde bucal e indicadores socioeconômicos municipais. Rev. saúde pública, v. 39, n. 6, p. 930-936, 2005.

FIGUEIREDO, Márcia Cançado et al.. Descrição da saúde bucal e de indicadores socioeconômicos de uma população adulta. Description of oral health and socioeconomic indicators of an adult population. DOI: 10.5212/Publ. Biologicas. v. 17i2. 0001. Publicatio UEPG: Ciências Biológicas e da Saúde, v. 17, n. 2, 2012.

GONÇALVES, Evelise Ribeiro; PERES, Marco Aurélio; MARCENES, Wagner. Cárie dentária e condições sócio-econômicas: um estudo transversal com jovens de 18 anos de Florianópolis, Santa Catarina, Brasil. Cad. Saúde Pública, v. 18, n. 3, p. 699-706, 2002.

GUARNIZO-HERREÑO, Carol C. et al.. Socioeconomic inequalities in oral health in different European welfare state regimes. Journal of epidemiology and community health, 2013. 
JUNQUEIRA, Simone Rennó et al.. Indicadores socioeconômicos e recursos odontológicos em municípios do Estado de São Paulo, Brasil, no final do século XX. Epidemiol Serv Saude, v. 15, n. 4, p. 41-53, 2006.

IPEADATA. Dados e indicadores sociais. Disponível em: http://www.ipeadata.gov.br/> .

THEÓPHILO, Carlos Renato; MARTINS, Gilberto de Andrade. Metodologia da investigação científica para ciências sociais aplicadas. São Paulo: Atlas, v. 2, 2009.

MINAYO, Maria Cecília; HARTZ, Zulmira Maria de Araújo.; BUSS, Paulo Marchiori. Qualidade de vida e saúde: um desafio necessário. Ciência e Saúde Coletiva, v. 5, n. 1, p. 2533, 2000.

PALMIER, Andréa Clemente et al. Socioeconomic indicators and oral health services in an underprivileged area of Brazil. Revista Panamericana de Salud Pública, v. 32, n. 1, p. 22 29, 2012.

SILVA, Luiz Anildo Anacleto et al.. Um olhar sócio-epidemiológico sobre o viver na sociedade atual e suas implicações para a saúde humana. Texto \& Contexto Enfermagem, v. 15, p. 170-177, 2006.

SHEIHAM, Aubrey. Oral health, general health and quality of life. Bulletin of the World Health Organization, v. 83, n. 9, p. 644-644, 2005.

SKUDUTYTE-RYSSTAD, Rasa; ERIKSEN, Harald M. Changes in caries experience among 35-year-old Oslo citizens, 1973-2003. Acta Odontologica, v. 65, n. 2, p.72-77, 2007. 